\title{
Methods of Reducing the Radiation Dose to the Pediatric Age Groups in Computed Tomography of Brain
}

\author{
S. Vidyasagar ${ }^{1}$, Abubacker Sulaiman $\mathrm{F}^{2}$ \\ ${ }^{1}$ Associate Professor, ${ }^{2}$ Professor, Department of Radiology, Dhanalakshmi Srinivasan Medical College and Hospital, Siruvachur, \\ Perambalur, India
}

Corresponding author: Dr.Vidyasagar, No:32, Rajresidency, Ramarayar agraharam, Tennur, Trichy, India

DOI: http://dx.doi.org/10.21276/ijcmsr.2019.4.1.31

How to cite this article: S. Vidyasagar, Abubacker Sulaiman F. Methods of reducing the radiation dose to the pediatric age groups in computed tomography of brain. International Journal of Contemporary Medicine Surgery and Radiology. 2019;4(1):A120-A123.

\section{A B S T R A C T}

Introduction: CT scan is essential diagnostic modality in diagnostic radiology. But the disadvantage is high radiation dose for both adult and pediatric age groups. Radiation dose depends on region of interest and scanner design. Therefore, the aim of this study was to assess the methods in reducing the radiation dose among pediatric age group while doing CT scan examination.

Material and Methods: A retrospective analysis of the radiation dose received by the patient was calculated by the formula DLP [DLP $=$ CTDI vol X L (DLP-Dose Length Product; CTDI-CT Dose Index; L-length of the table)]. Radiation dose was reduced by decreasing $\mathrm{mAs}, \mathrm{kVp}$ and increasing the pitch. Patients were imaged in CT Philips brilliance 6 and ingenuity core 128 slice.

Results: By increasing the pitch, there was reduction in $\mathrm{mAs}$ and $\mathrm{kVps}$, selecting approximate patient weight, a pronounced decrease in radiation dose was observed.

Conclusion: By the use of DLP calculation, change was observed in mAs and kVp followed by raised pitch and decreased FOV which ultimately leads to the effective reduction in dosage of the patient.

Keywords: Radiation Dose; KVP; Pediatrics; Mas, Computed Tomography

\section{INTRODUCTION}

CT scan was invented by G.N Hounsfield in the year of 1972 as a CAT scan (computed axial tomography). The term axial was used to differentiate from the conventional tomography technique. Then CT scan was updated with different types for reducing the radiation dose and the time duration.

CT scan is essential imaging modality in diagnostic radiology for both pediatrics and adults. The use of $\mathrm{CT}$ in pediatrics has significantly increased in recent times since it has greater diagnostic capabilities. CT creates images in cross-section by measurement of $\mathrm{x}$-ray attenuation properties of the body from different directions. CT radiation dose are comparatively higher than conventional radiography. There are potential risks in developing cancer when exposure to radiation crosses limits. The ionizing radiation doses delivered by CT are 100 to 500 times higher than the conventional radiography and are linked to an increased risk of cancer. ${ }^{1,2}$

Individuals in pediatric age groups are more sensitive to the ionizing radiation because their cells are cumulative and their long life span. There should be proper consideration when pediatric individuals are subjected to undergo CT scan. Radiation dose can be minimized to a certain extent technically by increasing $\mathrm{kVp}$ and decreasing $\mathrm{mAs}$, and limiting the scan to only the region suspected of any abnormality. Hence, optimization while performing a CT scan should be made necessary to avoid increased radiation dose. Although there are alternative modalities for pediatric imaging without radiation such as ultrasound and MRI, there are certain limitations due to which $\mathrm{CT}$ is considered. In ultrasound, there is no proper visualization of bones and bowel whereas in MRI, fracture in bone is not imaged clearly and time duration of scan is more. . $^{3,4}$

From previous literature, it was found that Brenner et al first assesses lifetime cancer risk induced by ionizing radiation exposure from pediatric CT. They revealed that 500 of the children who undergo $\mathrm{CT}$ each year would ultimately die of cancer attributable to ionizing radiation at $\mathrm{CT}$ on the basis of radiologic practices in the United States. All physicians and radiologists must first ensure the appropriateness of the clinical indication to avoid unnecessary scans, which is the important step for reducing the overall radiation exposure at $\mathrm{CT}^{5,6}$

After an appropriate clinical indication and justification have been approved, imaging settings can be customized to obtain the required clinical information at the lowest dose possible. Application of the scanning parameters used in adults to children must be avoided, because it leads to a substantial increase in the effective dose without providing additional benefits for diagnosis. If a single-phase scan can provide sufficient diagnostic information, avoidance of multiphase scanning such as non-enhanced CT allows for a drastic reduction in dose. The length of the scanning area on the body should be limited to the area of concern to eliminate unnecessary radiation. Various techniques such as automated 
exposure control, lowering tube current, tube voltage, highpitch acquisition and IR algorithms can help in reducing radiation dose or improving the image quality of low-dose pediatric CT. ${ }^{7,8,9}$

Radiation dose is particularly relevant in children because of the high sensitivity of dividing tissues to ionizing radiation and the long latent period of oncogenic effects as compared to adults. Thus, minimizing radiation dose is of particular importance in this context. In addition, children receive higher effective doses because of their smaller body size. For these reasons, the potential increase in cancer risk is of concern in children who require CT scans. ${ }^{10,11}$ Hence, the aim of the present study was to assess the method to reduce radiation dose in computed tomography of brain among paediatrics by changing machine parameters and scanning methods.

\section{MATERIAL AND METHODS}

The present study was a retrospective study which was conducted among pediatric patients who underwent CT examination from 01-02-2016 to 02-07-2016. Total 70 patients were scanned by Philips brilliance 6 and Ingenuity Core128 slice CT machine. Radiation dose received by the patient was calculated by the formula DLP -Dose Length Product. It was collected from PACS. Some were scanned with $350 \mathrm{mAs} / 120 \mathrm{kV}$, others were scanned with $250 \mathrm{mAs} / 100 \mathrm{kV}$. Only pediatric patients under 15 years of age were included in the study and uncooperative patients were excluded from the study.

The effective dose was calculated from the DLP (Dose length product). It is an approximation of the energy absorbed by the patient. Machine parameters that determine the radiation dose are: Decreasing the tube current (mAs), Decreasing beam energy $(\mathrm{kVp})$ and increasing the pitch.

\section{Tube current (mAs)}

The tube current determines the number of electrons accelerated across the $\mathrm{x}$-ray tube and the number of $\mathrm{x}$-ray produced. Tube current is given in units of $\mathrm{mA}$. Radiation dose is directly proportional to product of $\mathrm{mA}$ and the scan time per slice or $\mathrm{mAs}$. Since $\mathrm{mAs}$ depends on patient weight and clinical indication, it is decreased accordingly as more $\mathrm{mAs}$ will give more radiation.

\section{Beam energy $(\mathbf{k V p})$}

It determines the photon energy. By changing the $\mathrm{kVp}$, radiation dose changes proportional to the square of the percentage change in tube potential.

\section{Pitch}

Pitch is defined as the ratio of table top movement during one full rotation to collimator length.

The pitch increases the duration of scan time which also decreases the radiation dose. The disadvantage is image clarity will also decrease.

\section{STATISTICAL ANALYSIS}

Data collected was compiled in Microsoft office excel sheet and further analysed by SPSS version 21 . The descriptive analysis was performed using percentages and was represented in the form of graphs.

\section{RESULTS}

In the present study, out of 70 subjects, 43 subjects were scanned with the help of $350 \mathrm{mAs}$ and $120 \mathrm{kVp}$ and 27 were scanned with the help of 200-300 mAs. It was seen that subjects who were scanned with the help of $350 \mathrm{mAs}$ and $120 \mathrm{kVp}$, the DLP was found above the reference level and those with 200-300 mAs, the DLP was equal to reference level (Figure 1, 2 and 3).

Graph no. 1 showed that for 0 to 5 years group, it was observed $64 \%$ of people received more radiation than the reference level and only $36 \%$ received within the reference level. Graph

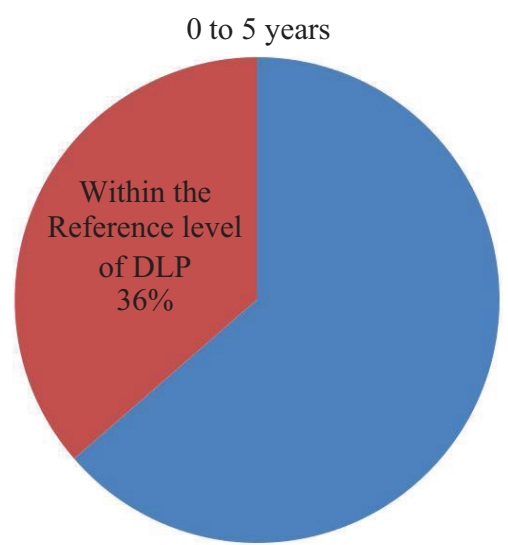

Graph-1: shows the distribution of data based on DLP among $0-5$ years

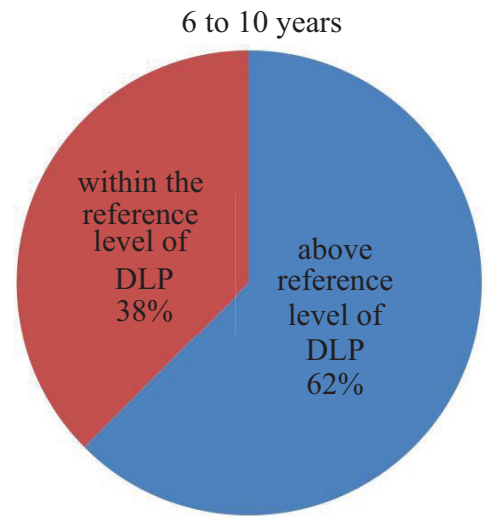

Graph-2: shows the distribution of data based on level of DLP among 6 to 10 years

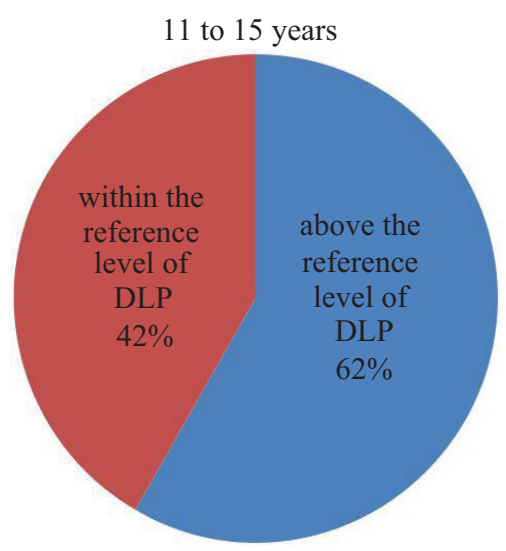

Graph-3: shows the distribution of data based on DLP among 11 to 15 years 
DLP in 250-300 mAs and $120 \mathrm{kvp}$

DLP in $350 \mathrm{mAs}$ and $120 \mathrm{kvp}$

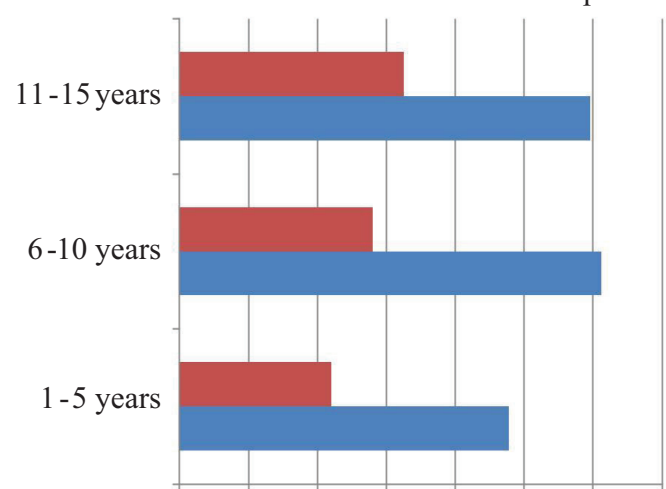

Graph-4: shows the difference in DLP among different age groups

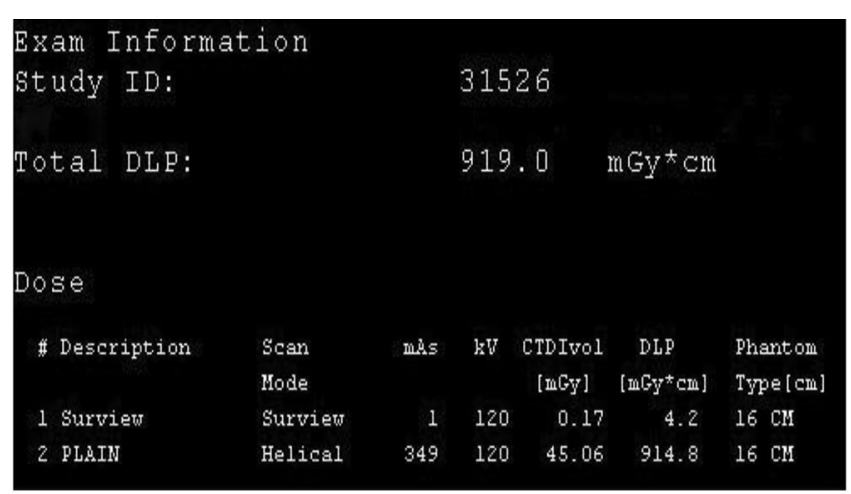

Figure no. 1 shows the $120 \mathrm{kV}$ and $350 \mathrm{mAs}$ with DLP of 919

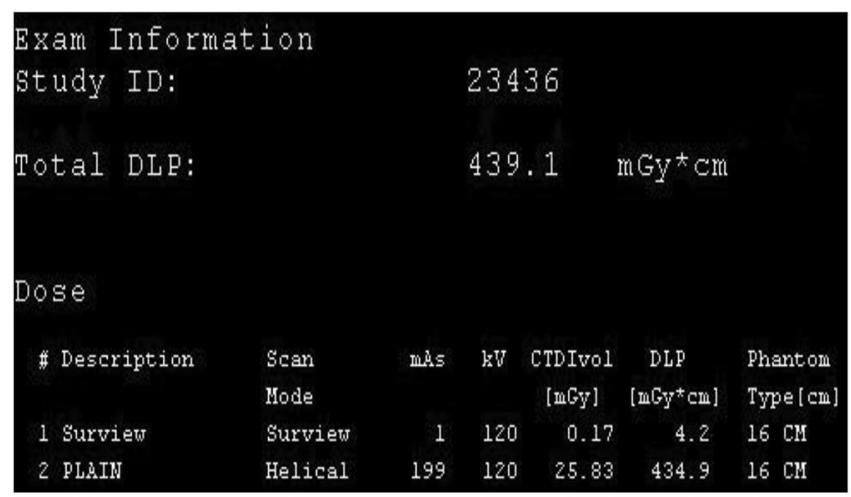

Figure no. 2 shows the $200 \mathrm{mAs}$ and $120 \mathrm{kV}$ with Total DLP of 439

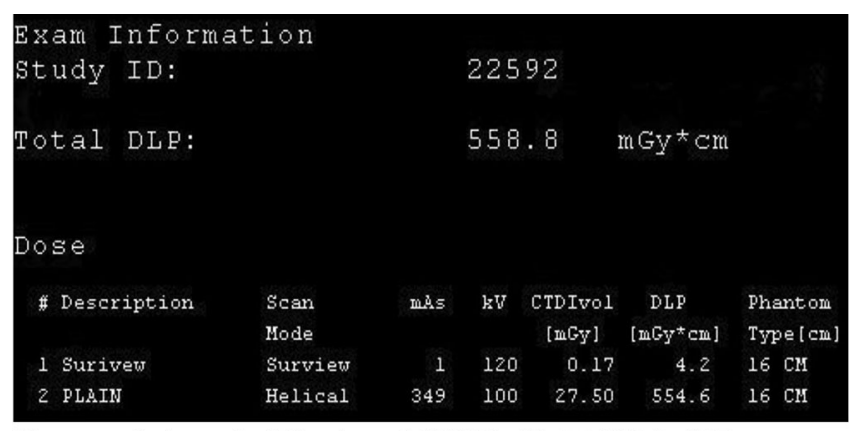

Figure no. 3 shows the $349 \mathrm{mAs}$ and $120 \mathrm{kV}$ with total DLP of 55

no. 2 showed that for 6 to 10 years, it was observed that $62 \%$ of people received more radiation than the reference level and only $38 \%$ received within the reference level. Graph no.
3 showed that In 11 - 15years group, 58\% people received more radiation then the reference level and $42 \%$ of people received within the reference level. Graph no. 4 showed that the children scanned with DLP of $350 \mathrm{mAs}$ and $120 \mathrm{kVp}$ received more radiation dose than the reference value. Also, children with DLP of $250-300 \mathrm{mAs}$ and $120 \mathrm{kVp}$ received radiation within the reference value.

\section{DISCUSSION}

Several factors should be taken in to account when $\mathrm{kVp}$ is decreased because lowering the $\mathrm{kVp}$ means $\mathrm{mAs}$ has to be increased and when selecting weight from a range, $\mathrm{kVp} /$ $\mathrm{mAs} /$ dose technique varies accordingly lower $\mathrm{kVp}$ increases the scan times because of $\mathrm{mAs}$ limit which thus increase the motion artifact. But, irrespective of patient presentation the protocols were used in such a way that radiation significantly decreased without affecting the image quality.

From the patient's perspective, benefit in the use of CT is more than the small radiation received. However there are few cases in which patient may require second or third CT scan which increases the patient radiation dose to certain extent. Thus, the use of variable machine parameters is the valid requirement for reduction of radiation dose.

Lower tube voltage has an advantage over the conventional voltages of $120-140 \mathrm{kVp}$ in terms of cerebral gray-white matter contrast for non-enhanced brain CT scan. This is because the photoelectric component for gray matter is 4-6\% higher than that of white matter and the gray matter attenuation is higher than the white matter attenuation at lower $\mathrm{kVp}$, which can lead to an improved gray-white matter contrast-to-noise ratio using a low $\mathrm{kVp} \mathrm{CT}$ scan.

In a recent study, Ben- David et al demonstrated that unenhanced head CT scanning at a low tube voltage $(80 \mathrm{kVp})$ delivers a higher gray matter-white matter contrast-to-noise ratio per dose compared to scans performed using a tube voltage of $120 \mathrm{kVp}$ in adults. Similar to this previous study in adults, our study also revealed a statistically significant increase, from 2.18 to $2.35(\mathrm{P}=0.019)$, of gray matter-white matter contrast-to-noise ratio with $80-\mathrm{kVp} \mathrm{CT.}{ }^{12}$

In a study by Ji Eun Park et al, a significant reduction in radiation dose was achieved with $120-\mathrm{kVp}$ CT scans, whilst providing a similar overall image quality to that achieved with $120-\mathrm{kVp}$ CT scans. The mean effective dose from 80$\mathrm{kVp}$ CT scans was $6.5 \%$ lower than that observed for 120 $\mathrm{kVp}$ CT scans. Lowering tube voltage is inevitably associated with an increase in image noise. Even though the increased tube current to compensate for the higher noise level at 80 $\mathrm{kVp}$, the measured noise values were still greater with the $80-\mathrm{kVp}$ CT scans than those observed in the $120-\mathrm{kVp}$ CT scans. ${ }^{13}$

Furthermore, the gray-white matter contrast-to-noise ratio was still increased at $80-\mathrm{kVp}$ CT despite the increased noise value, and there was no significant difference in overall image quality scores between $80-$ and $120-k V p$ scans. Secondly, various kinds of surgical materials and devices may introduce substantial beam hardening artifacts (e.g., streak or metallic artifacts), which are often more severe at lower tube voltage than at high tube potential.

There were a few limitations to our study. Firstly, scans were 
acquired at different times. Simultaneous acquisition of both scans would have enabled more accurate comparison of image quality parameters. Secondly, the study population was relatively small and the disease states were not evaluated due to the small and heterogeneous study population.

\section{CONCLUSION}

With the help of DLP method, reduction in radiation dosage was achieved among children.

\section{REFERENCES}

1. Miglioretti DL, Johnson E, Williams A, et al. The use of computed tomography in pediatrics and the associated radiation exposure and estimated cancer risk. JAMA Pediatr 2013; 167(8):700-707.

2. Pearce MS, Salotti JA, Little MP, et al. Radiation exposure from $\mathrm{CT}$ scans in childhood and subsequent risk of leukaemia and brain tumours: a retrospective cohort study. Lancet 2012; 380(9840):499-505.

3. Mathews JD, Forsythe AV, Brady Z, et al. Cancer risk in 680,000 people exposed to computed tomography scans in childhood or adolescence: data linkage study of 11 million Australians. BMJ 2013; 346(3):f2360.

4. Huang WY, Muo CH, Lin CY, et al. Paediatric head $\mathrm{CT}$ scan and subsequent risk of malignancy and benign brain tumour: a nation-wide population-based cohort study. Br J Cancer 2014; 110(9):2354-2360.

5. Udayasankar UK, Braithwaite K, ArvanitiMet al. Lowdose nonenhanced head CT protocol for follow-up evaluation of children with ventriculoperitoneal shunt: reduction of radiation and effect on image quality. AJNR Am J Neuroradiol 2008; 29(2):802-806.

6. Krille L, Dreger S, Schindel R, et al. Risk of cancer incidence before the age of 15 years after exposure to ionising radiation from computed tomography: results from a German cohort study. Radiat Environ Biophys 2015; 54(1):1-12. [Published correction appears in Radiat Environ Biophys 2017; 56(3):293-297.]

7. Journy N, Roué T, Cardis E, et al. Childhood CT scans and cancer risk: impact of predisposing factors for cancer on the risk estimates. J Radiol Prot 2016;36(1):N1-N7.

8. Berrington de Gonzalez A, Salotti JA, McHugh K, et al. Relationship between paediatric CT scans and subsequent risk of leukaemia and brain tumours: assessment of the impact of underlying conditions. $\mathrm{Br} \mathrm{J}$ Cancer 2016; 114(4):388-394.

9. Meulepas JM, Ronckers CM, Smets AM, et al.Leukemia and brain tumors among children after radiation exposure from CT scans: design and methodological opportunities of the Dutch Pediatric CT Study. Eur J Epidemiol 2014; 29(4):293-301.

10. Thierry-Chef I, Dabin J, Friberg EG, et al. Assessing organ doses from paediatric CT scans-a novel approach for an epidemiology study (the EPI-CT study). Int J Environ Res Public Health 2013; 10(2):717-728.

11. Walsh L, Shore R, Auvinen A, Jung T, Wakeford R. Risks from CT scans-what do recent studies tell us? J Radiol Prot 2014; 34(1):E1-E5.

12. Ben-David E, Cohen JE, Nahum Goldberg S et al. Significance of enhanced cerebral gray-white matter contrast at $80 \mathrm{kVp}$ compared to conventional 120
$\mathrm{kVp}$ CT scan in the evaluation of acute stroke. J Clin Neurosci 21:1591-1594.

13. Wakeford R. The risk of childhood leukaemia following exposure to ionising radiation-a review. J Radiol Prot 2013; 33(1):1-25.

\section{Source of Support: Nil; Conflict of Interest: None}

Submitted: 09-01-2018; Accepted: 17-02-2018; Published online: 30-03-2019 